\title{
Treatment of Primary Intra-Articular Pigmented Villonodular Synovitis of the Knee by Using Complete Arthroscopic Synovectomy and Postoperative Low-Dose Radiotherapy
}

Kai Tie

Wuhan University Zhongnan Hospital

Hua Wang

Wuhan University Zhongnan Hospital

Biao Chen

Wuhan University Zhongnan Hospital

Xu Yang

Wuhan University Zhongnan Hospital

Liaobin Chen ( $\square$ lbchen@whu.edu.cn )

Zhongnan Hospital of Wuhan University

\section{Research article}

Keywords: pigmented villonodualr synovitis, arthroscopic synovectomy, radiation therapy, knee

Posted Date: October 21st, 2020

DOI: https://doi.org/10.21203/rs.3.rs-93182/v1

License: (c) (i) This work is licensed under a Creative Commons Attribution 4.0 International License.

Read Full License 


\section{Abstract}

\section{Background}

Pigmented villonodular synovitis (PVNS) is a locally aggressive, proliferative synovial tissue disorder, the optimal treatment regimen for diffuse PVNS is still undefined. The aim of this study was to evaluate the long-term clinical outcome of the treatment of complete arthroscopic synovectomy combined with lowdose external radiotherapy in the knee affected by primary intra-articular diffuse PVNS.

Methods

A series of 18 patients diagnosed with primary diffuse PVNS by clinical and pathological examination were enrolled in this study from May 2009 to January 2016. All patients underwent complete arthroscopic synovectomy and postoperative local low-dose (20 Gy) external radiotherapy, and were evaluated before treatment and final follow-up using the criteria of Ogilvie-Harris and International Knee Documentation Committee (IKDC) score.

Results

The patients were followed-up for a mean of 68 months (35 to 120 months). No recurrence was found. No surgical and radiation-related complications were observed. The mean score according to OgilvieHarris criteria was 3.19 which rated as poor, and the IKDC function score was $37.1 \pm 3.8$ before treatment. At the final follow-up, the mean score of Ogilvie-Harris was 8.79 which rated as good, and the IKDC score was $83.9 \pm 11.3(p<0.05)$.

\section{Conclusion}

Treatment for primary intra-articular diffuse PVNS in the knee with complete arthroscopic synovectomy and postoperative low-dose external radiotherapy had good clinical outcome with no complication and recurrence.

\section{Background}

Pigmented villonodular synovitis (PVNS) is a locally aggressive, proliferative synovial tissue disorder [1]. It affects the synovial lining of joints, tendon sheaths and bursas, and may invade and destroy surrounding soft tissue and bone. The annual incidence has been calculated as 1.8 cases per million population [2]. PVNS may be intra- or extra-articular, and is classified by clinical presentation and biological behavior as localized or diffuse [3]. Diffuse forms are mainly intra-articular and affect the knee joint [4]. The presenting symptoms of diffuse PVNS are mainly spontaneous swelling, diffuse nonspecific knee pain, and a decreased range of motion, which are more likely to imitate some chronic inflammatory states [5]. The early diagnosis is difficult owing to the non-specific presentation and slow symptom progression, intervals between first signs and diagnosis are long: 10 months to 3 years [6]. 
Because of the extremely low incidence of PVNS and the long delays before local recurrence, the optimal treatment regimen for diffuse PVNS is still undefined [7].

Management of primary diffuse PVNS of the knee is difficult, because the local recurrence rate is high with whatever treatment strategy [8]. The reported different treatment combinations included surgical synovectomy with or without adjuvant therapy $[9,10]$. Surgical treatment for diffuse PVNS of the knee involves open or arthroscopic synovectomy, which one is more appropriate is still open for debate. Open synovectomy entails a greater risk of knee stiffness, and longer hospital stay and rehabilitation [11]. Advantages of arthroscopic synovectomy include quicker recovery time, less intraoperative blood loss, less postoperative stiffness, and improved access to the posterior compartments [12]. A systematic review concluded that no difference in local recurrence between open and arthroscopic total synovectomy in the surgical treatment of diffuse PVNS, and a lower rate of postoperative complications after arthroscopic synovectomy [7]. Therefore, the arthroscopic approach for total synovectomy could be recommended for diffuse PVNS when technically possible.

The aim of treatment of diffuse PVNS is to remove all abnormal synovial tissue to relieve pain, lower the risk of joint destruction, and avoid local recurrence. Successful arthroscopic treatment of diffuse PVNS of the knee requires meticulous excision of all diseased synovial tissue [13]. Incomplete synovectomy alone for diffuse PVNS should be avoided because of a high risk of local recurrence [7]. Total arthroscopic synovectomy should include anterior compartment, medial and lateral gutters and posterior compartment. Two or three standard portals are established for anterior compartment and medial and lateral gutters, posteromedial and posterolateral accessory portals are established for posterior compartment of the knee $[1,12]$. Researches demonstrated that good clinical outcomes had been acquired by using the arthroscopic synovectomy for diffuse PVNS with abovementioned portals $[1,14]$. Thus, we used two posteromedial portals to permit complete posteromedial synovectomy.

Radiation therapy is the most widely used adjuvant treatment, it could reduce recurrence in diffuse PVNS [8]. However, there are few studies showing dose-response relationships for postoperative radiation therapy in patients with PVNS. Because PVNS is a benign entity [6], one research reported that low-dose (20 Gy) external radiotherapy appears to be as effective as moderate-dose treatment (around $35 \mathrm{~Gy}$ ) [15]. The purpose of this retrospective study was to evaluate the clinical outcome and recurrence of complete arthroscopic synovectomy associated with low-dose external-beam radiotherapy in the knee affected by primary intra-articular diffused PVNS.

\section{Methods}

\section{Study cohort}

Patients with diffuse PVNS, as confirmed on pathological evaluation, who underwent complete arthroscopic synovectomy and external-beam radiotherapy between May 2009 and January 2016, were enrolled into the study. All patients gave informed written consent and the study was approved by the Ethical Committee of Zhongnan Hospital of Wuhan University. The inclusion criteria were: clinical 
symptoms and MRI findings of diffuse PVNS; postoperative pathologic confirmation of primary diffuse PVNS; no major degenerative changes of the knee. The exclusion criteria were: diffuse PVNS with extraarticular extension into bone and soft tissue or need open surgery; recurrent diffuse PVNS; PVNS in any other joints than the knee.

18 patients were enrolled in total, 8 were men and 10 were women. The mean age of the patients was 38.3 years (19 to 63). Presenting symptoms were mainly spontaneous swelling, diffuse nonspecific knee pain, and a decreased range of motion. X-rays of the affected knee were performed to detect and exclude other pathologies such as osteoarthritis. All patients were subjected to an MRI scan for better preoperative evaluation (Figure 1). All of the procedures described were performed by the same surgical team comprising all the authors. The team not only provided preoperative and perioperative care to the patients but also participated in postoperative recovery.

\section{Surgical techniques}

The patient was supine and a thigh tourniquet was used under spinal anaesthesia. A $30^{\circ}$ arthroscope (Linvatec) and a $5.5 \mathrm{~mm}$ motorized shaver were routinely used. Standard anteriolateral and anteriomedial portals were made. After the examination of the anterior compartment, the complete synovectomy was done including anteromedial and medial gutter, notch area, anterolatearal and lateral gutter, and medial and lateral submeniscal area (Fig. 2). When the popliteal tendon hiatus area was difficult to access, extreme lateral portal was established to facilitate the synovectomy; Then two posteromedial portals were created to finish the synovectomy of posteromedial compartment; the high portal $2.5 \mathrm{~cm}$ to $3 \mathrm{~cm}$ above the joint line and the low portal level with the joint line. The arthroscope was introduced through the high posteromedial portal, providing a clear view. A power-shaver was inserted through the low posteromedial portal to debride the synovium of posteriomedial compartment (Fig. 3). Then the mid-third of the posterior septum was removed until the posteriolateral compartment could be visualized clearly. Moving the arthroscope through the posterior septum, the posterolaral portal was made and the posterolateral synovectomy was accomplished (Fig. 4). Finally, the suprapatellar pouch region was completely debrided and lateral suprapatellar portal was used if needed. Arthroscopic electroablation was done just when an oozing vessel seen. An intra-articular suction drain was sustained 24 hours after surgery. The suture was removed from the surgical wounds around 2 weeks after the operation.

\section{Rehabilitation protocol}

The patients began physical therapy after removing the drain. Straight leg raising and ankle pump were started from the first post-operative day. Passive range of motion exercises with a continuous passive motion was done in the first postoperative two weeks, and then changed to active exercises. Weight bearing was allowed according patient's tolerance. Therapeutic goals were set to achieve maximum range of motion, restore quadriceps muscle strength, and decrease swelling and pain in the early postoperative period.

\section{Radiotherapy}


In 4-6 weeks postoperatively, all patients underwent local adjuvant radiotherapy applied with a 6-MV linear accelerated photon beam. The total dose was $20 \mathrm{~Gy}$, which was performed 10 times, once every other day. Fractioned irradiation doses were delivered to the anterior and posterior fields of the operated knee.

\section{Follow-up and evaluation}

After radiotherapy, patients were re-examined MRI and then re-evaluated by the same radiologist every 6 months. Each patient was evaluated before treatment and at the final follow-up visit, the evaluation criteria used in this study was according to Ogilvie-Harris [16]. The criteria comprise four parameters: articular pain, synovitis or articular effusion, range of motion, and functional ability. Each of the four parameters is graded on a scale of 0 to 3 points. To globally evaluate the results of the combined treatment, we calculated the arithmetic average of the scoring obtained by the various parameters considered in homogeneous classes of treatment. The patient's condition was rated as excellent (scores between 10 and 12), good (scores between 7 and 9), fair (scores between 4 and 6), or poor (scores between 0 and 3). Because the parameter of functional ability in this evaluation criteria is non-specific, we used the International Knee Documentation Committee (IKDC) [17] scores to evaluate the knee function. In the process of the follow-up, recurrence case was diagnosed on the basis of renewed onset of clinical symptoms and MRI screening, and was confirmed pathohistologically.

\section{Statistical analysis}

The preoperative and final follow-up scores of Ogilvie-Harris criteria and IKDC scores were statistically analyzed using SPSS 17.0 software (SPSS Inc., Chicago, Illinois). The Mann-Whitney U test was used compare the IKDC scores. A $p$ value $<0.05$ was considered statistically significant.

\section{Results}

All 18 patients were available for follow-up. Mean follow-up was 68 months (range from 35 to 120 months). The mean time from symptom onset to surgery was 9 months (range from 3 to 29 months), the most frequent preoperative symptoms were joint effusion, diffuse nonspecific knee pain, and a decreased range of motion. Four patients reported a history of trauma. Outerbridge I or II cartilage lesions were found in five patients, severe erosions in meniscus were found in two patients. The mean evaluation score according to the Ogilvie-Harris criteria before treatment was 3.19, which corresponded to a rating of "poor". The mean score after treatment was 8.79, rated as "good". The IKDC score of all patients increased significantly from $37.1 \pm 3.8$ before treatment to $83.9 \pm 11.3$ after treatment $(p=0.001)$. No recurrence was noted at final follow up.

There were no complications during or after the arthroscopic procedure. No evidence of swelling, infection, joint stiffness, and neurovascular lesions were detected. None of these patients experienced radiation-related early or late complications such as skin reactions, poor wound healing, joint stiffness 
and radiation-induced sarcomas. Three patients who had preoperative cartilage lesions had pain when squatting.

\section{Discussion}

With the reason of low morbidity and the difficulty of diagnosis, it is lack of prospective comparative studies about the management of diffuse PVNS. No current consensus exists as to the optimum treatment. In the present study, 18 patients presenting with primary intra-articular diffuse PVNS of the knee were treated with total arthroscopic synovectomy combined with low-dose of external-beam radiotherapy, and were followed-up at a mean period of 68 months. No recurrence was found in this case series at final follow up. Previous clinical studies demonstrated that the interval between initial treatment and recurrence ranged from 1 month to 20 years, with a mean of 4.9 years [18-21]. The follow-up time in our study was longer than this mean recurrence time, the recurrence rate was better than the prior reports which varied from $8 \%$ to $46 \%$ with all treatment methods $[14,16,19,22]$. The score of Ogilvie-Harris criteria showed good evaluated result. The total arthroscopic synovectomy and low-dose of externalbeam radiotherapy might play a major role in our acquired clinical outcome, and the other reason may be the included patient presenting with primary intra-articular diffuse PVNS.

Arthroscopic surgery offers the possibility to resect diseased synovium with minimal loss of function and faster recovery. Total excision of the synovium is considered the basis of treatment to prevent local recurrence of diffuse PVNS [3, 23]. The arthroscopic approach for total synovectomy should be recommended when technically possible. The decision whether to operate arthroscopically is made according to the MRI findings of the extent to which the knee is affected. Patients with diffuse PVNS confined only within the knee could be chosen for arthroscopy by an experienced surgeon, because skills using posterior portals need time and practice to develop, even for a seasoned arthroscopist. Incomplete synovectomy for diffuse PVNS should be avoided because of a high risk of local recurrence [7], it is very important for surgeons to improve and upgrade their skills in arthroscopic surgery to be able to use it to the fullest. There are several essential points for the successful use of total arthroscopic synovectomy: (1) Visualization and access of the posterior compartments should be maximized, remove the mid-third of the posterior septum and use the trans-septum approach to examine the poster compartment; (2) Submeniscal areas, especially lateral submeniscal areas, are easy to miss debriding. The popliteal tendon hiatus area is difficult to access, sometimes extreme lateral portal is needed to finish the synovectomy; (3) In terms of the order of synovectomy, excision of the suprapatellar pouch region should be the last step, because the synovectomy in this region can cause the extravasation of lavage solution in anterior knee, which may limit the flexion of knee in the operation.

Radiation therapy has been an alternative mode of treatment in PVNS for many years [22, 24]. A metaanalysis by Mollon et al concluded that synovectomy combined with perioperative radiotherapy for diffuse PVNS was associated with a reduced rate of recurrence ${ }^{8}$. The adjuvant radiotherapy contains external and intra-articular modes, however the optimal dose of radiation for treatment is still unknown. The usual used dose of external radiotherapy was $20-50 \mathrm{~Gy}$ in existing literatures $[15,25]$. And the 
radiotherapy also has potential complications such as skin reactions, poor wound healing, joint stiffness and radiation-induced sarcomas [1]. Therefore, in the present study, we used external radiotherapy of lowdose (20 Gy), which was much lower than the other studies, with no recurrence and no complication. The mean postoperative IDKC score of the affected knees was 83.9 which demonstrated that the knee function was not influenced by the low-dose radiation therapy. The type of PVNS included in our study was primary intra-articular diffuse PVNS, some literatures suggested that total synovectomy is sufficient for this type [7,13], adjuvant radiotherapy treatment is not necessary. In our opinion, postoperative radiotherapy is obligatory, because meticulous total synovectomy is just for macroscopic view, the microscopic pathology may still exist, thus radiotherapy is an optimal complemental treatment under this circumstance. That was also the reason why no recurrence was noted in our case series. Low-dose of radiation was sufficient to kill remaining diseased cells, and not enough to cause radiation-related complications.

Although satisfying result had been obtained in our study, there were several limitations. First, inclusion of patients with primary intra-articular diffuse PVNS of the knee limited further extrapolation beyond the cases studied. Second, this study was a retrospective series without comparison to a control group, it might have introduced selection bias arise from some factors related to both surgeons and patients. Third, only a limited number of patients were studied. Due to the low incidence of PVNS, it is almost impossible to perform large-scale prospective studies.

\section{Conclusion}

The outcome of this study proved that total arthroscopic synovectomy combined with low-dose external radiotherapy is optimal for treating primary intra-articular diffuse PVNS of the knee joint. It could be safely and reliably used with minimal complications, fast post-operative recovery and satisfactory control of recurrence rates.

\section{Abbreviations}

PVNS: pigmented villonodular synovitis; IKDC: International Knee Documentation Committee.

\section{Declarations}

\section{Acknowledgements}

We thank Dr. A. Gibson for his contribution to the language editing of the manuscript.

\section{Funding}

This work was financially supported by National Natural Science Foundation of China (No. 81702159) and National health and family planning scientific research project (No. 2016ZX-01-018S06) 
Availability of data and materials

All data generated or analyzed in this study were available from the corresponding author through emails on reasonable request.

\section{Authors' contributions}

Kai Tie designed the study, acquired data and drafted the manuscript; Hua Wang acquired data, and drafted the manuscript; Biao Chen analyzed data and revised the manuscript; Xu Yang analyzed and interpreted data and drafted the manuscript; Liaobin Chen conceived and designed this study, drafted and revised the manuscript.

\section{Ethics approval and consent to participate}

This study was approved by the ethics committee of the Zhongnan Hospital of Wuhan University. Informed consent was obtained from all the participants.

\section{Consent for publication}

Written informed consent was obtained from each patient to authorize the publication of their images and pathologic documentation. All authors read and approved the final manuscript and consented to publication.

\section{Competing interests}

The authors declare that they have no competing interests.

\section{References}

1. Kubat O, Mahnik A, Smoljanović T, Bojanić Arthroscopic Arthroscopic treatment of localized and diffuse pigmented villonodular synovitis of the knee. Coll Antropol. 2010; 34(4):1467-72.

2. Myers BW, Masi AT. Pigmented villonodular synovitis and tenosynovitis: a clinical epidemiologic study of 166 cases and literature review. Medicine (Baltimore). 1980; 59(3): 223-38.

3. Granowitz SP, D'Antonio J, Mankin HL. The pathogenesis and long-term end results of pigmented villonodular synovitis. Clin Orthop Relat Res. 1976; (114): 335-51.

4. Botez P, Sirbu PD, Grierosu C, Mihailescu D, Savin L, Scarlat MM. Adult multifocal pigmented villonodular synovitis--clinical review. Int Orthop. 2013; 37(4): 729-33.

5. Dines JS, DeBerardino TM, Wells JL, et al. Long-term follow-up of surgically treated localized pigmented villonodular synovitis of the knee. Arthroscopy. 2007; 23(9): 930-7.

6. Gouin F, Noailles T. Localized and diffuse forms of tenosynovial giant cell tumor (formerly giant cell tumor of the tendon sheath and pigmented villonodular synovitis). Orthop Traumatol Surg Res. 2017; 103(1S): S91-S97. 
7. Auregan JC, Klouche S, Bohu Y, Lefève N, Herman S, Hardy P. Treatment of pigmented villonodular synovitis of the knee. Arthroscopy. 2014; 30(10): 1327-41.

8. Mollon B, Lee A, Busse JW, et al. The effect of surgical synovectomy and radiotherapy on the rate of recurrence of pigmented villonodular synovitis of the knee: an individual patient meta-analysis. Bone Joint J. 2015; 97-B: 550-7.

9. Blanco CE, Leon HO, Guthrie TB. Combined partial arthroscopic synovectomy and radiation therapy for diffuse pigmented villonodular synovitis of the knee. Arthroscopy. 2001; 17(5): 527-31.

10. Chin KR, Barr SJ, Winalski C, Zurakowski D, Brick GW. Treatment of advanced primary and recurrent diffuse pigmented villonodular synovitis of the knee. J Bone Joint Surg Am. 2002; 84(12): 2192-202.

11. Gu HF, Zhang SJ, Zhao C, Chen Y, Bi Q. A comparison of open and arthroscopic surgery for treatment of diffuse pigmented villonodular synovitis of the knee. Knee Surg Sports Traumatol Arthrosc. 2014; 22(11): 2830-6.

12. De Ponti A, Sansone V, Malcherè Result of arthroscopic treatment of pigmented villonodular synovitis of the knee. Arthroscopy. 2003; 19(6): 602-7.

13. Kramer DE, Frassica FJ, Frassica DA, Cosgarea AJ. Pigmented villonodular synovitis of the knee: diagnosis and treatment. J Knee Surg. 2009; 22(3): 243-54.

14. Keyhani S, Kazemi SM, Ahn JH, Verdonk R, Soleymanha M. Arthroscopic Treatment of Diffuse Pigmented Villonodular Synovitis of the Knee: Complete Synovectomy and Septum RemovalMidterm Results. J Knee Surg. 2019; 32(5): 427-33.

15. Park G, Kim YS, Kim JH, et al. Low-dose external beam radiotherapy as a postoperative treatment for patients with diffuse pigmented villonodular synovitis of the knee: 4 recurrences in 23 patients followed for mean 9 years. Acta Orthop. 2012; 83(3): 256-60.

16. Ogilvie-Harris DJ, McLean J, Zarnett ME. Pigmented villonodular synovitis of the knee: The results of total arthroscopic synovectomy, partial arthroscopic synovectomy and arthroscopic local excision. J Bone Joint Surg Am 1992; 74(1): 119-23.

17. Irrgang JJ, Ho H, Harner CD, Fu FH. Use of the International Knee Documentation Committee guidelines to assess outcome following anterior cruciate ligament reconstruction. Knee Surg Sports Traumatol Arthrosc. 1998; 6(2): 107-14.

18. Byers PD, Cotton RE, Deacon OW, et al. The diagnosis and treatment of pigmented villonodular synovitis. J Bone Joint Surg Br. 1968; 50(2): 290-305.

19. Flandry FC, Hughston JC, Jacobson KE, Barrack RL, McCann SB, Kurtz DM. Surgical treatment of diffuse pigmented villonodular synovitis of the knee. Clin Orthop Relat Res. 1994; (300):183-92.

20. Chiari C, Pirich C, Brannath W, Kotz R, Trieb K. What affects the recurrence and clinical outcome of pigmented villonodular synovitis? Clin Orthop Relat Res. 2006; 450: 172-8.

21. Schwartz HS, Unni KK, Pritchard DJ. Pigmented villonodular synovitis. A retrospective review of affected large joints. Clin Orthop Relat Res. 1989; (247): 243-55. 
22. Sharma V, Cheng EY. Outcomes after excision of pigmented villonodular synovitis of the knee. Clin Orthop Relat Res. 2009; 467(11): 2852-8.

23. Flandry F, Hughston JC. Pigmented villonodular synovitis. J Bone Joint Surg Am 1987; 69: 942-9.

24. Zvijac JE, Lau AC, Hechtman KS, Uribe JW, Tjin ATEW. Arthroscopic treatment of pigmented villonodular synovitis of the knee. Arthroscopy 1999; 15(6): 613-7.

25. Griffin AM, Ferguson PC, Catton CN, et al. Long-term outcome of the treatment of high-risk tenosynovial giant cell tumor/pigmented villonodular synovitis with radiotherapy and surgery. Cancer. 2012; 118(19): 4901-9.

\section{Figures}




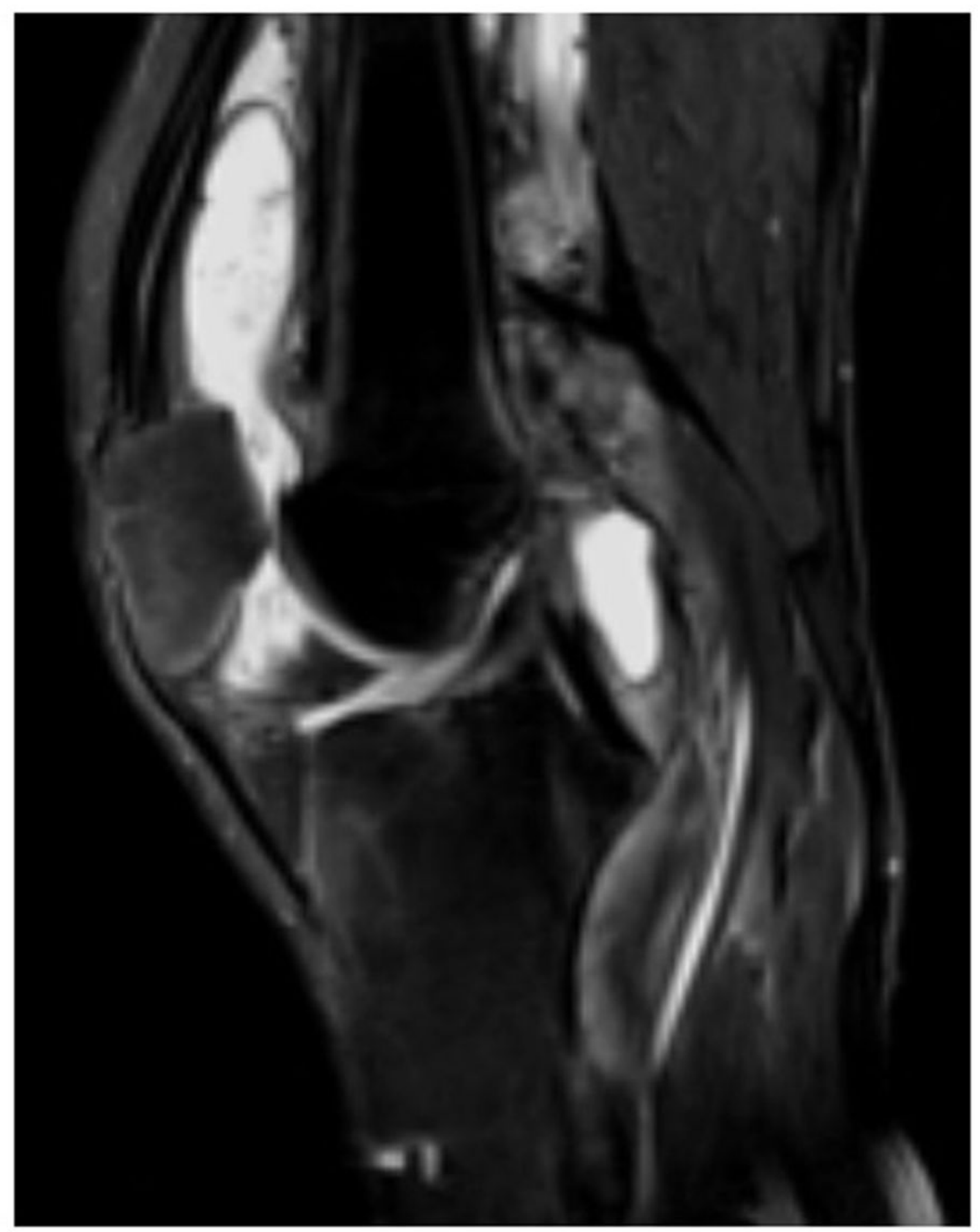

Figure 1

Preoperative magnetic resonance imaging. 


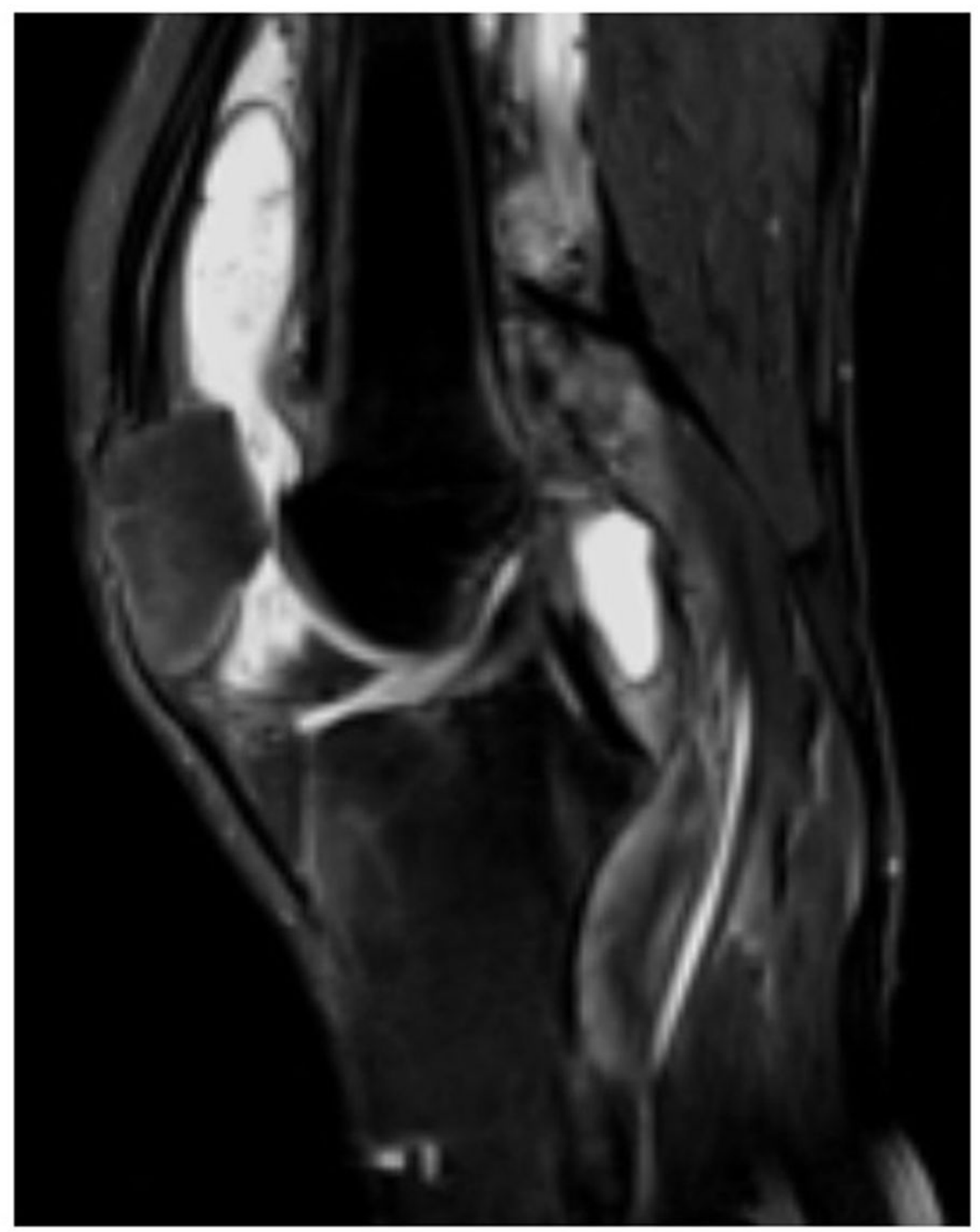

Figure 1

Preoperative magnetic resonance imaging. 


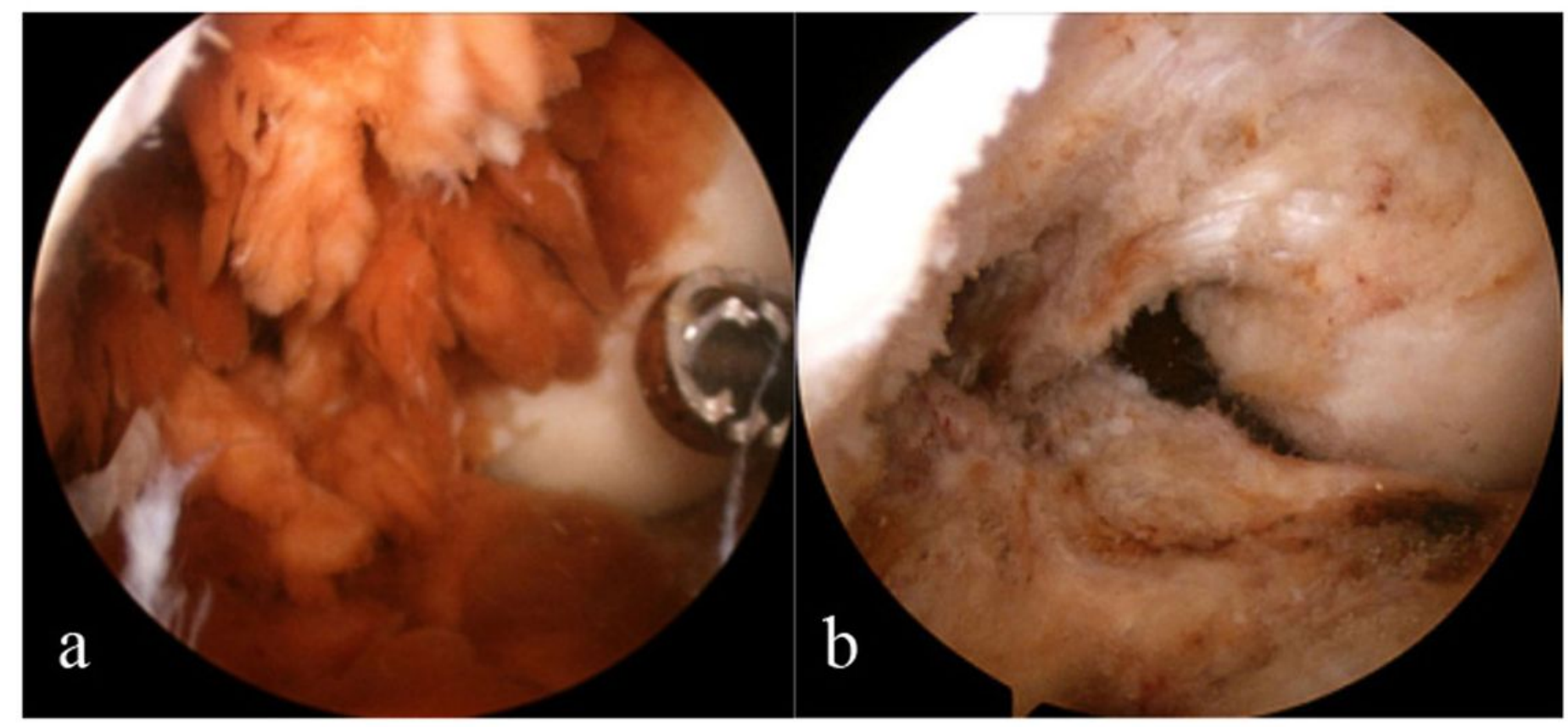

Figure 2

Anterior compartment viewing. (A) before synovectomy; (B) after synovectomy.

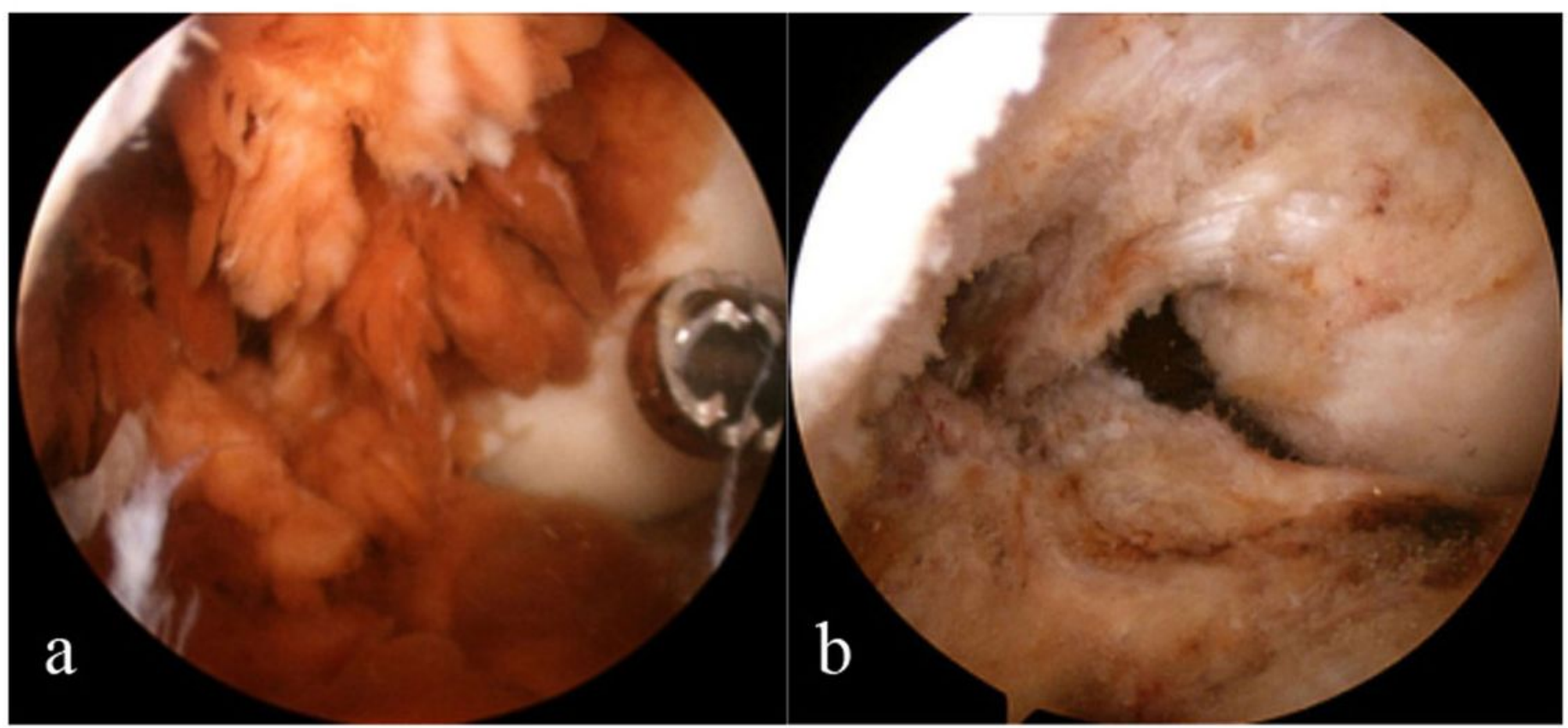

Figure 2

Anterior compartment viewing. (A) before synovectomy; (B) after synovectomy. 


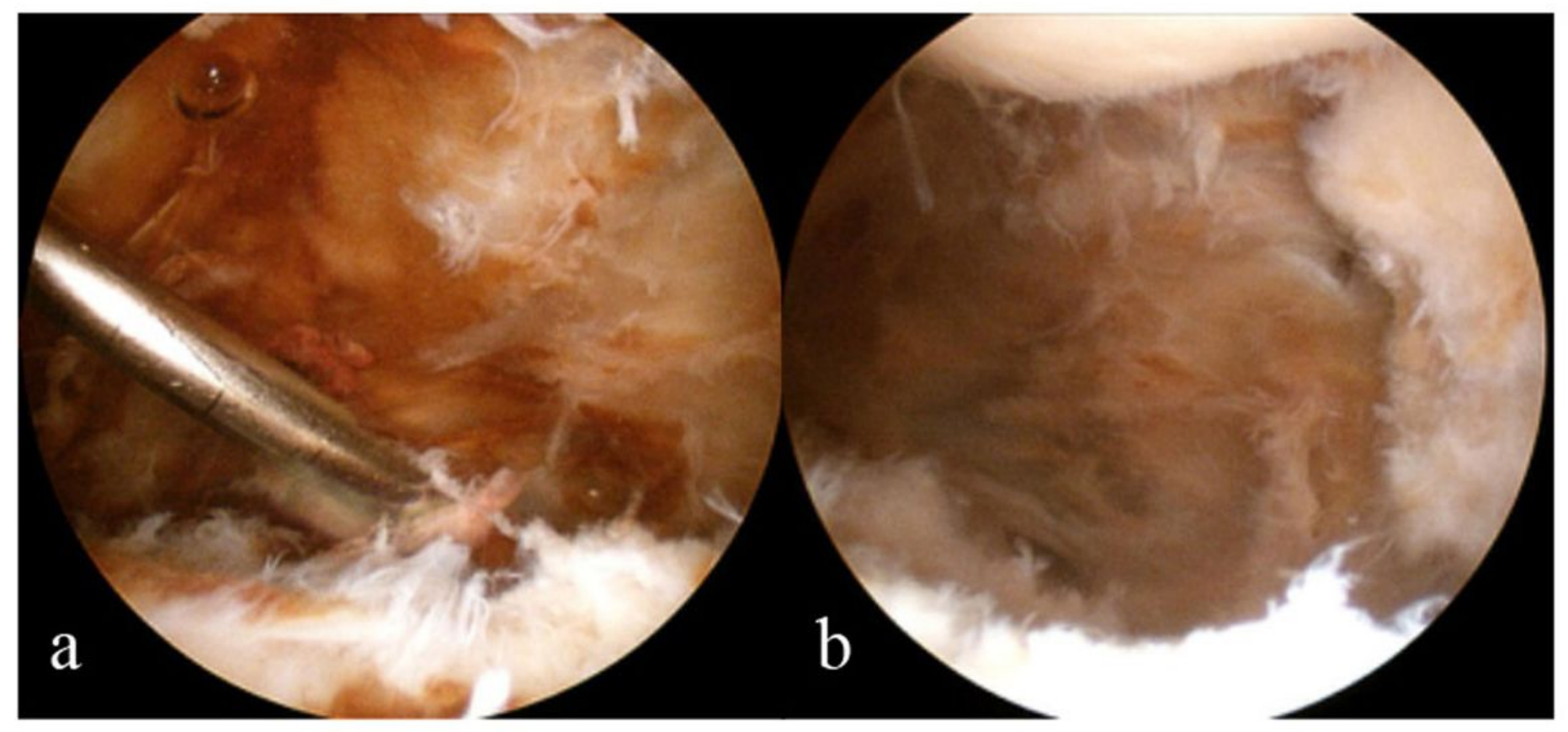

Figure 3

Posteromedial compartment viewing. (A) before synovectomy; (B) after synovectomy.

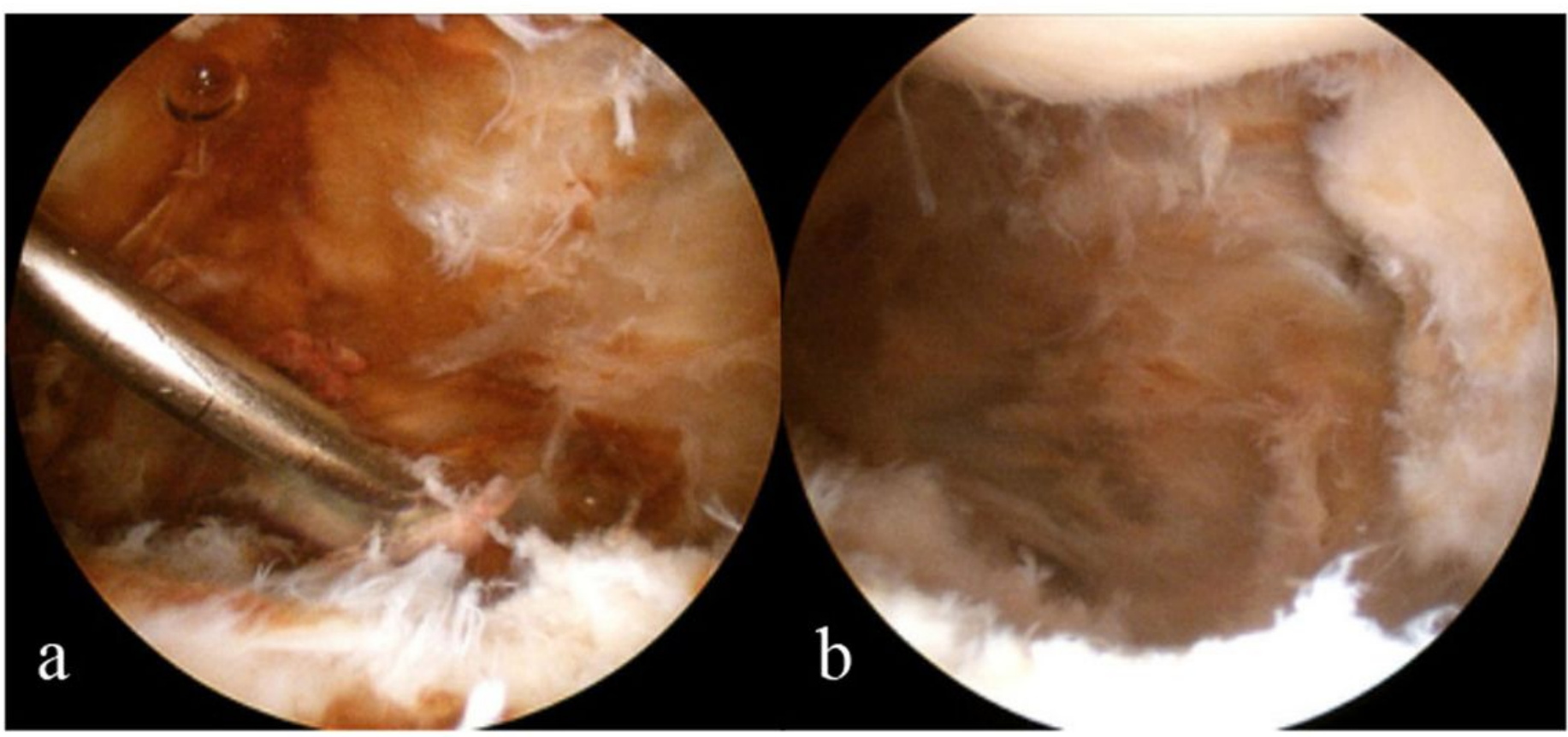

Figure 3

Posteromedial compartment viewing. (A) before synovectomy; (B) after synovectomy. 


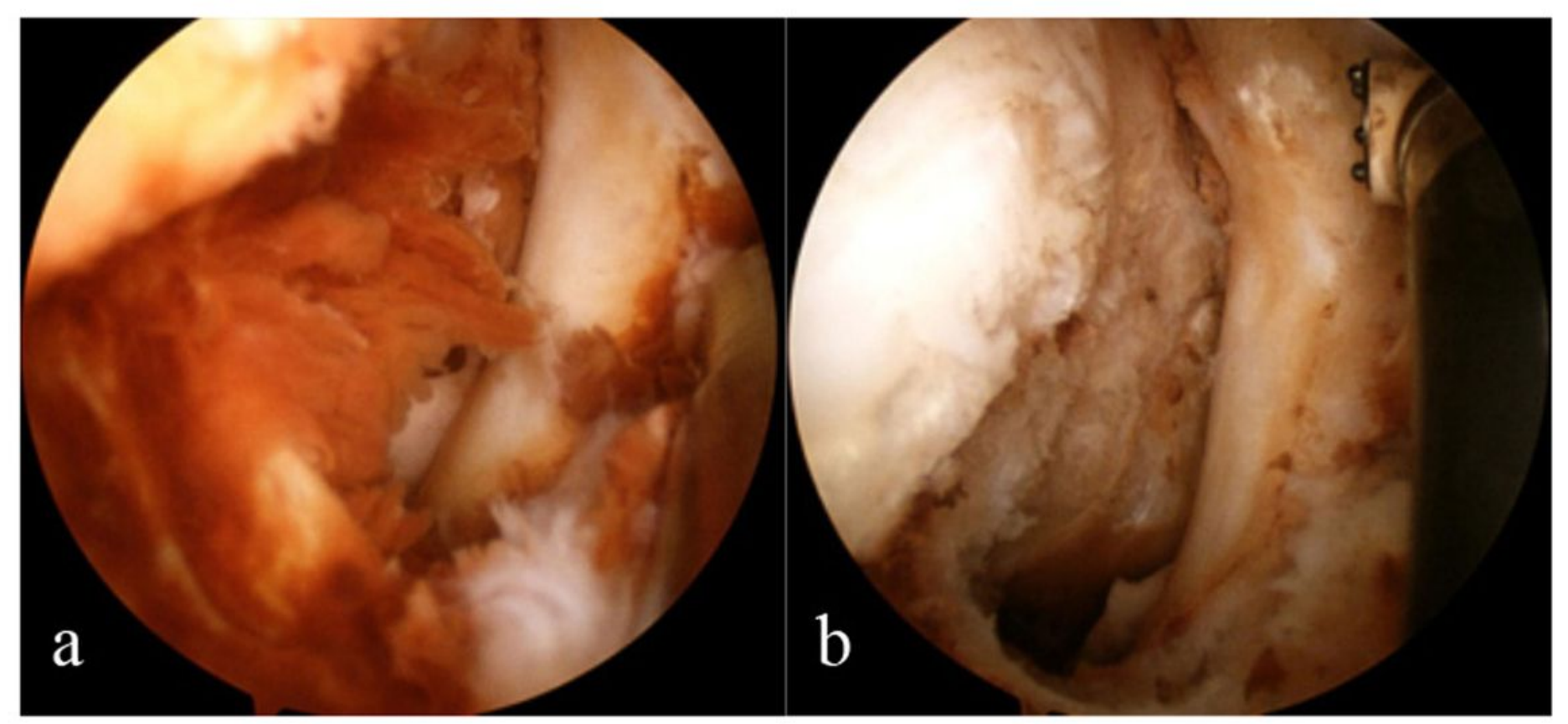

\section{Figure 4}

Posterolateral compartment viewing. (A) before synovectomy; (B) after synovectomy.

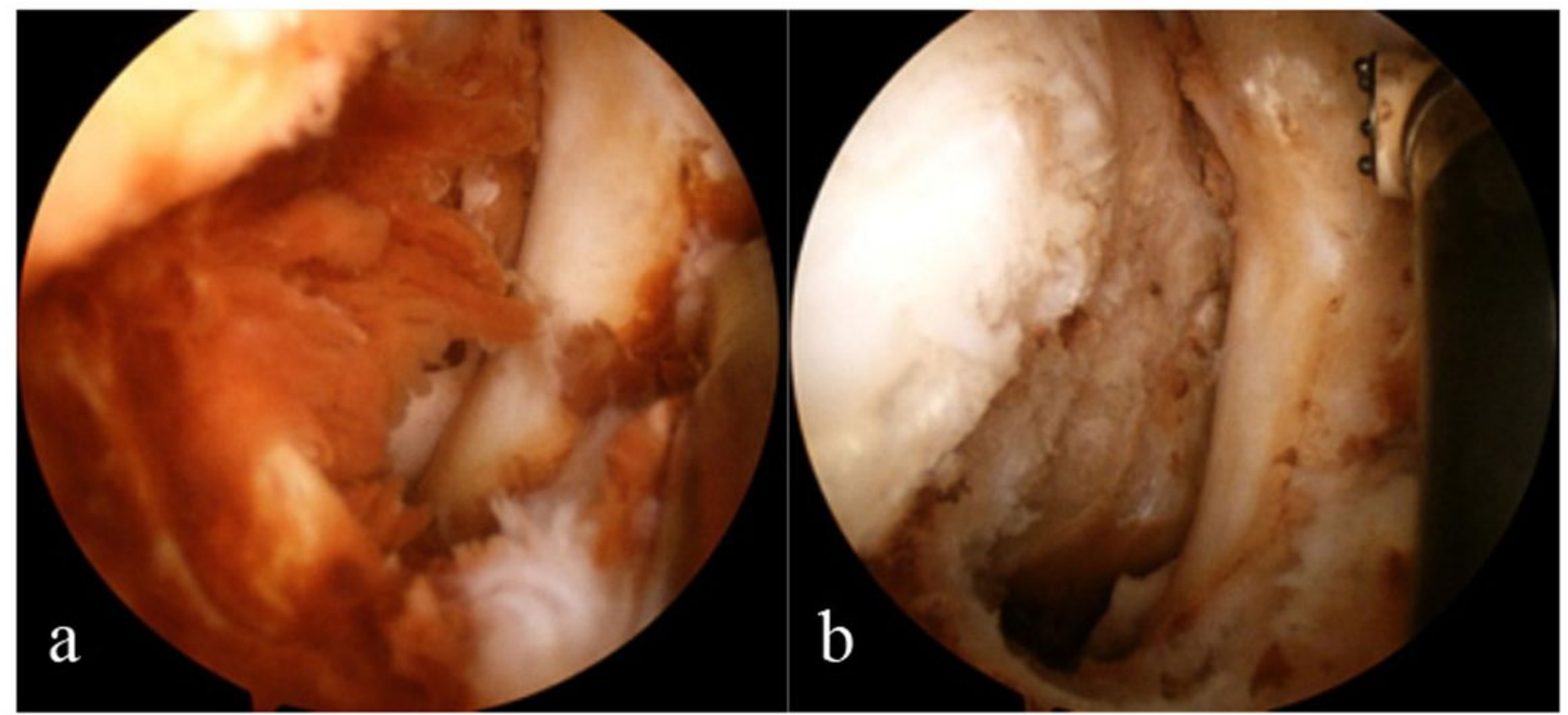

Figure 4

Posterolateral compartment viewing. (A) before synovectomy; (B) after synovectomy. 